\title{
Infliximab Versus Adalimumab in Patients with Biologic-Naïve Crohn's Disease: Is the Difference Real?
}

\author{
Tamara Mogilevski ${ }^{1}$. Miles P. Sparrow ${ }^{1,2}$ \\ Published online: 8 February 2018 \\ ○) Springer Science+Business Media, LLC, part of Springer Nature 2018
}

Since their introduction almost 20 years ago, anti-tumor necrosis factor agents (anti-TNFs) have revolutionized Crohn's disease (CD) management. Despite the availability of newer classes of biologic therapies, anti-TNFs remain the most effective agents for the treatment of moderate-severe luminal and fistulising CD [1-4]. Infliximab and adalimumab are the two most commonly used anti-TNFs in clinical practice worldwide. Although randomized controlled trials comparing different classes of IBD therapies have recently been proposed, large prospective head-to-head comparative effectiveness studies of anti-TNF agents have not been performed and are unlikely to be performed unless funded by non-pharmaceutical sources. Therefore, to date there have only been small, retrospective analyses, predominantly including anti-TNF exposed patients, comparing the efficacy of anti-TNF agents. As such, clinical decisions regarding the choice of anti-TNF used are often based on issues of logistics and convenience, rather than on scientific evidence. The significance of this lack of scientific rigor in therapeutic decision-making should not be underestimated considering the extremely high financial costs incurred by the use of biologic agents.

To help address this issue, Benmassaoud et al. reporting in this issue of Digestive Diseases and Sciences [5] performed a retrospective audit at McGill University Health Centre comparing clinical outcomes at 12 months between anti-TNF naïve $C D$ patients treated with infliximab and adalimumab. Two hundred and twenty patients (143 infliximab and 77 adalimumab) were followed for almost 5 years (infliximab-58.6 months, adalimumab-44.6 months); the largest cohort of anti-TNF naïve patients with the longest period

Miles P. Sparrow

m.sparrow@alfred.org.au

1 Department of Gastroenterology, The Alfred Hospital, 55 Commercial Road, Melbourne 3004, Australia

2 Faculty of Medicine, Nursing and Health Sciences, Monash University, Melbourne, Australia of follow-up reported to date. The primary endpoint was clinical response and remission at 12 months, as assessed by the Harvey-Bradshaw index. Secondary endpoints included corticosteroid-free remission, durable remission, adverse events, treatment failure, and outcomes of combination therapy versus biologic monotherapy. As is acknowledged, the infliximab and adalimumab groups were mismatched, a potential and flaw inherent in any retrospective study. Infliximab patients were younger, more commonly female, had a shorter disease duration prior to anti-TNF commencement, and had a longer observation time. More importantly, infliximab patients had more perianal disease and were more likely to be receiving corticosteroid therapy at the time of anti-TNF initiation. Given that the choice of anti-TNF used was at the discretion of the clinician, this mismatching may reflect real-world decision-making where clinicians may choose to use infliximab in "sicker" patients.

Rates of clinical remission and corticosteroid-free remission at 12 months were similar between groups: 63.8 versus $76.3 \%,(p=0.139)$ and 54.1 versus $44.7 \%,(p=0.354)$, respectively, for infliximab and adalimumab; in reassuring similarity to smaller studies [6-10]. Some subtle differences regarding secondary endpoints were noted. There was a trend to higher clinical response (but not remission) rates (91.2 vs. $78.9 \%, p=0.051)$ and lower $\mathrm{C}$-reactive protein levels ( 4.0 vs. $14.9, p=0.058$ ) in adalimumab patients at 12 months. Although these findings may reflect mismatching of groups with the potential for selection bias, they were retained after a sensitivity analysis was performed. In contrast, discontinuation of therapy was more common in infliximab patients ( 58.0 vs. $32.5 \%(p<0.001)$, likely in part due to more adverse events ( 36.1 vs. $15.5 \%, p=0.006$ ), and in particular infusion-/injection-site reactions (17.5 vs. $6.5 \%, p=0.024)$. Importantly, combination therapy with an immunomodulator led to significantly higher rates of clinical remission at 12 months compared to monotherapy in the infliximab group ( 81.2 vs. $52.1 \%, p=0.008)$ but not in the adalimumab group ( 81.2 vs. $70.4 \%, p=0.328)$. 
Benmassaoud et al.'s study adds to the growing body of evidence that the two most commonly used anti-TNF $\alpha$ agents have similar clinical efficacy in "real-world" use. This is counterintuitive to the belief that perhaps infliximab is more "potent" due to its intravenous administration and weight-based dosing schedule. This perception is even illustrated in this study by the selection bias of clinicians in choosing infliximab over adalimumab in patients with more severe disease activity (requiring corticosteroids) or phenotypes (perianal disease), mirroring the bias observed in other similarly designed retrospective studies [6, 7].

The finding that combination therapy with an immunomodulator is superior with infliximab but not with adalimumab was also reported in a recent multicentre study from the Australian and New Zealand Inflammatory Bowel Disease Consortium [11.] The benefits of combination therapy are probably due to both the dual mechanisms of action of each agent and the improved pharmacokinetics of the biologic agent with the addition of an immunomodulator. This pharmacokinetic benefit was confirmed in a recent post hoc analysis of the Study of Immunomodulator Naiive Patients in Crohn's Disease (SONIC) cohort that showed that while combination therapy did increase serum anti-TNF concentrations, for a given drug concentration clinical outcomes where similar regardless of the use of combination therapy or monotherapy [12]. This suggests that the pharmacokinetic benefits of combination therapy may be the most important explanation for improved clinical outcomes. Of note, in this study only $30 \%$ of patients were simultaneously receiving a thiopurine and $12 \%$ methotrexate; the small sample sizes for these subgroup analyses urge caution in the interpretation of these results.

Some methodological study flaws warrant mentioning. Primary non-response rates in this study were surprisingly low; $7 \%$ for infliximab and $6.5 \%$ for adalimumab, possibly due in part to the retrospective study design and the use of subjective disease activity indices, rather than objective biomarkers as study endpoints. The mismatching of groups, as has been summarized, is also an inherent potential weakness of retrospective studies, but the sensitivity analysis attempts to address this. Although the study population contains a significant number of patients with perianal disease (56 in the infliximab group and 18 in the adalimumab group), no subanalysis of perianal disease outcomes was provided, a key omission given that it has only been addressed in a few prior studies, with conflicting results. Furthermore, although just over $40 \%$ of patients in each group underwent anti-TNF dose intensification, no information is given regarding the indication, strategy, duration, or outcomes of dose intensification. The need for dose intensification was also not included in the sensitivity analysis. Given the well-recognized need for dose optimization, ideally followed by subsequent de-escalation once remission is attained, in a significant subgroup of patients receiving anti-TNFs, this information is important for both clinical and economic reasons. Finally, no information regarding therapeutic drug monitoring is given, whether in reference to the need for dose intensification, or overall study outcomes.

The main strengths of this study are the large cohort size of anti-TNF naïve patients and the long duration of monitoring. The study should provide reassurance to clinicians by adding to the growing body of literature suggesting that the two most commonly used anti-TNF $\alpha$ agents - infliximab and adalimumab - are largely equivalent in efficacy in Crohn's disease in "real-world" clinical practice.

The study brings up a number of issues for future research. Firstly, it again raises questions regarding the role of combination therapy in the setting of adalimumab, compared to infliximab. Future studies addressing this clinically important question should incorporate therapeutic drug monitoring to confirm whether the pharmacokinetic advantages of combination therapy justify its additional risks in adalimumab-treated patients. Secondly, the question of whether the two anti-TNF $\alpha$ agents are equivalent in efficacy against perianal disease remains unclear. Again, future studies in this area should include pre-specified perianal disease-specific outcome measures including the use of medical imaging. The use of therapeutic drug monitoring in perianal disease also warrants investigation, as it appears this subgroup of patients may require higher serum drug concentrations to attain remission compared to patients with luminal disease [13]. Personalization of therapy with dose intensification followed by de-escalation, again in part based on therapeutic drug monitoring, should be incorporated in future studies. The use of harder objective endpoints in the form of endoscopy, fecal biomarkers, and cross-sectional imaging will also produce more robust and reliable results in future studies.

\section{References}

1. Hanauer SB, Feagan BG, Lichtenstein GR, et al. Maintenance infliximab for Crohn's disease: the ACCENT I randomised trial. Lancet. 2002;359:1541-1549.

2. Sands BE, Anderson FH, Bernstein CN, et al. Infliximab maintenance therapy for fistulizing Crohn's disease. $N$ Engl J Med. 2004;350:876-885.

3. Sandborn WJ, Hanauer SB, Rutgeerts P, et al. Adalimumab for maintenance treatment of Crohn's disease: results of the CLASSIC II trial. Gut. 2007;56:1232-1239.

4. Colombel JF, Schwartz DA, Sandborn WJ, et al. Adalimumab for the treatment of fistulas in patients with Crohn's disease. Gut. 2009;58:940-948.

5. Benmassaoud A, Al-Taweel T, Sasson MS, et al. Comparative effectiveness of infliximab versus adalimumab in patients with biologic-Naïve Crohn's disease. Dig Dis Sci. (Epub ahead of print). https://doi.org/10.1007/s10620-017-4874-6. 
6. Zorzi F, Zuzzi S, Onali S, et al. Efficacy and safety of infliximab and adalimumab in Crohn's disease: a single centre study. Aliment Pharmacol Ther. 2012;35:1397-1407.

7. Varma P, Paul E, Huang C, Headon B, Sparrow MP. A retrospective comparison of infliximab versus adalimumab as induction and maintenance therapy for Crohn disease. Intern Med J. 2016;46:798-804.

8. Osterman MT, Haynes K, Delzell E, et al. Comparative effectiveness of infliximab and adalimumab for Crohn's disease. Clin Gastroenterol Hepatol. 2014;12:811-7.e3.

9. Narula N, Kainz S, Petritsch W, et al. The efficacy and safety of either infliximab or adalimumab in 362 patients with antiTNF- $\alpha$ naïve Crohn's disease. Aliment Pharmacol Ther. 2016;44:170-180.

10. Kestens C, van Oijen MG, Mulder CL, et al. Adalimumab and infliximab are equally effective for Crohn's disease in patients not previously treated with anti-tumor necrosis factor- $\alpha$ agents. Clin Gastroenterol Hepatol. 2013;11:826-831.

11. Doecke JD, Hartnell F, Bampton P, et al. Infliximab vs. adalimumab in Crohn's disease: results from 327 patients in an Australian and New Zealand observational cohort study. Aliment Pharmacol Ther. 2017;45:542-552.

12. Colombel JF, Adedokun OJ, Gasink C, et al. Hanauer SB higher levels of infliximab may alleviate the need of azathioprine comedication in the treatment of patients with Crohn's disease: a Sonic post hoc analysis. Gastroenterology. 2017;152:S37-S38.

13. Yarur AJ, Kanagala V, Stein DJ, et al. Higher infliximab trough levels are associated with perianal fistula healing in patients with Crohn's disease. Aliment Pharmacol Ther. 2017;45:933-940. https://doi.org/10.1111/apt.13970. 